\section{Prevalence and characteristics of vagus nerve variations on neck ultrasonography}

\author{
Dongbin Ahn', Gil Joon Lee', Jin Ho Sohn', Jeong Kyu Kim² \\ 'Department of Otolaryngology-Head and Neck Surgery, School of Medicine, Kyungpook \\ National University, Daegu; ${ }^{2}$ Department of Otolaryngology-Head and Neck Surgery, Catholic \\ University of Daegu School of Medicine, Daegu, Korea
}

Purpose: We aimed to evaluate the true prevalence and characteristics of vagus nerve (VN) variations using the carotid artery (CA) and the internal jugular vein (C-I axis).

Methods: We examined patients who underwent neck ultrasonography (US) conducted by a single operator. A VN variation was defined as a VN located anterior or medial to the C-I axis. The subtypes of VN variation were classified as anterolateral, anteromiddle, anteromedial, and medial based on the relative location of the $\mathrm{VN}$ to the CA. The primary outcome parameters were the prevalence of $\mathrm{VN}$ variations and differences according to side, age, and sex.

Results: Out of 536 patients, right and left VN variations were identified in $20(3.7 \%)$ and 186 $(34.7 \%)$, respectively $(\mathrm{P}<0.001)$. The anteromiddle type was the commonest type observed on both sides. Eight right (1.5\%) and 50 left VNs (9.3\%) were located $<2 \mathrm{~mm}$ from the lateral border of the ipsilateral thyroid gland $(\mathrm{P}<0.001)$. The prevalence of $\mathrm{VN}$ variations in male and female patients was $42.1 \%$ and $32.7 \%$, respectively $(P=0.029)$, and that in patients aged $<20$, $20-39,40-59$, and $\geq 60$ years was $23.8 \%, 22.5 \%, 34.4 \%$, and $47.4 \%$, respectively $(P<0.001)$. Conclusion: Variations in the VN position were relatively common on US. The variations primarily involved the left VN in the lower cervical region, and an increasing prevalence with age was observed.

Keywords: Neck; Vagus nerve; Variation; Ultrasonography; Prevalence

Key points: Overall prevalence of vagus nerve (VN) variation was $36.2 \%$, with a significant leftside predominance. VN variations were identified primarily in the lower cervical region (level IV). Prevalence of VN variation significantly increased from the 5th decade, with a linear increasing trend according to increasing age.

\section{Introduction}

The cervical portion of the vagus nerve (VN) is located within the carotid sheath, usually posterolateral to the carotid artery (CA) and posteromedial to the internal jugular vein (IJV) [1-3]. However, several variations in the VN position have been reported and the presence of these anatomical variations is of great importance [1-3]. From a surgical perspective, carotid endarterectomy is a leading cause of VN injuries, with a reported incidence of $1.5 \%-35.0 \%$ [2-5]. In addition, the VN is commonly manipulated during neck dissection along with the CA and the IJV, and the VN is also close to the
ULTRA

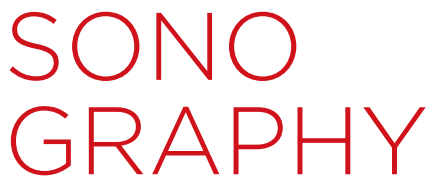

\section{ORIGINAL ARTICLE}

https://doi.org/10.14366/usg.21045 pISSN: 2288-5919 • eISSN: 2288-5943

Ultrasonography 2022;41:124-130

Received: February 25, 2021

Revised: April 25, 2021

Accepted: April 26, 2021

Correspondence to:

Dongbin Ahn, MD, Department

of Otolaryngology-Head and

Neck Surgery, School of Medicine,

Kyungpook National University, 130

Dongdeok-ro, Jung-gu, Daegu 41944, Korea

Tel. +82-53-200-5781

Fax. +82-53-423-4524

E-mail: godlikeu@naver.com
This is an Open Access article distributed under the terms of the Creative Commons Attribution NonCommercial License (http://creativecommons.org/ licenses/by-nc/4.0/) which permits unrestricted noncommercial use, distribution, and reproduction in any medium, provided the original work is properly cited.

Copyright (C) 2022 Korean Society of Ultrasound in Medicine (KSUM)

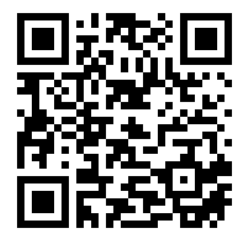

How to cite this article:

Ahn D, Lee GJ, Sohn JH, Kim JK. Prevalence and characteristics of vagus nerve variations on neck ultrasonography. Ultrasonography. 2022 Jan;41(1):124-130. 
surgical field of thyroidectomy [6]. Moreover, ethanol/radiofrequency ablation is commonly performed for the management of various neck masses, including thyroid, lymph node, and congenital lesions, and the $V N$ can be injured during these procedures $[2,7-10]$. Therefore, knowledge of anatomical variations in the course of the VN is paramount to reduce the risk of VN injury during both surgical and non-surgical management of various diseases of the cervical region.

To date, a few studies have described multiple types of VN variations on ultrasonography (US), including anterior and medial variations or anterior, medial, and posterior variations, and reported prevalence rates of $5.5 \%-37.8 \%[1-3,11]$. However, in these previous studies, it was unclear whether the variation was present at specific cervical regions or throughout the course of the cervical VN (from level II to level IV). In addition, all previous studies on VN position defined the presence of a variation based on the relative location of the VN to the CA alone. However, the VN is located within the carotid space together with the CA and the IJV; thus, a definition based on the CA alone seems to have limited clinical significance because the presence of a variation does not indicate that the VN is located at the superficial aspect of the carotid space. For example, even when the VN is located anterior to the CA, if the $\mathrm{VN}$ is overlaid by the IJV, the VN may be less vulnerable, as the IJV protects it (Fig. 1). Therefore, it is more reasonable to define variations of the $\mathrm{VN}$ position based on a reference axis consisting of both the CA and IJV (C-I axis), rather than using its relative location to the $\mathrm{CA}$ alone. In addition, when reporting VN variations, information about the specific cervical levels where a VN variation

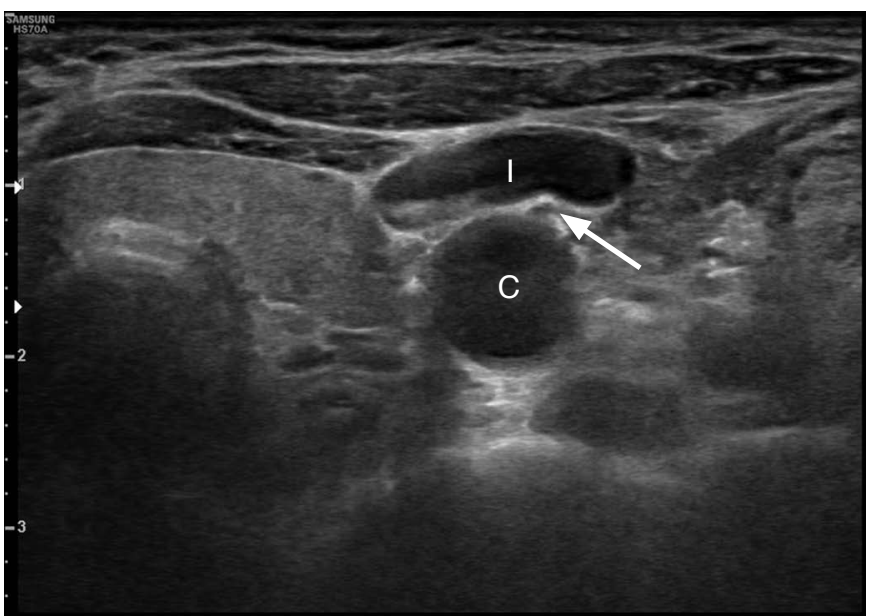

Fig. 1. Variations in the position of the vagus nerve showing no clinical significance. Although the vagus nerve (arrow) is located anterior to the carotid artery $(C)$, it is not at risk because it is covered and protected by the internal jugular vein (I). is present should be also included to provide clinically useful information that reduces the risk of VN injury during surgery or radiological interventions.

This study aimed to evaluate the true prevalence and characteristics of VN variations on neck US based on the reference C-I axis, in order to provide clinically useful information on cervical VN variations.

\section{Materials and Methods}

\section{Compliance with Ethical Standards}

The institutional review board of our hospital approved the study protocol (Kyungpook National University Hospital, 201701009), and written informed consent was obtained from all patients. The study was conducted in accordance with the ethical principles of the Declaration of Helsinki.

\section{Study Design and Patient Eligibility}

This study was designed as a prospective cohort study. The inclusion criteria of the present study were patients who underwent neck US by a single US operator for various indications, including evaluation of the thyroid, salivary gland, lymph node, and other neck lumps. The exclusion criteria included (1) patients who had a neck mass that infiltrated the carotid space, disrupting the normal structure of the carotid space; (2) patients who had a neck mass that involved the $\mathrm{VN}$, such as vagal schwannoma or metastatic carcinoma invading the $\mathrm{VN}_{\text {; }}(3)$ patients who had a previous history of neck surgery or irradiation; and (4) patients who underwent US examinations by other operators.

\section{Sample Size Calculation}

Based on the $5.5 \%-37.8 \%$ prevalence of $\mathrm{VN}$ variations in previous studies, to calculate the sample size, we defined the expected prevalence of $\mathrm{VN}$ variations in our study as $5.5 \%$, the precision as $2.25 \%$ (half of the expected prevalence), and the confidence interval as $95 \%[2,3,11]$. Using these values, the calculated sample size was 395 patients [12]. With this sample size as the lower limit of the study population, we enrolled eligible participants during the planned study period.

\section{Patient Position for US Examination}

In previous studies, VN position was evaluated in the supine position with neck extension, but without neck rotation [2,3]. However, most operations or procedures requiring precautions to prevent VN injury are performed with the patient in the supine position with extension and contralateral rotation of the neck. Therefore, all participants of 
the present study were placed in the supine position with extension and contralateral rotation of the neck for US examinations. However, in light of the possibility that this patient position might affect the VN position, we preliminarily evaluated whether the VN position changed after neck rotation in 50 patients and confirmed that the VN position was not affected by neck rotation.

\section{US Examinations and the Definition of VN Position}

All US examinations were performed in the outpatient department by a single operator who had 13 years of experience in US examinations of the head and neck regions. An HS 70A US device (Samsung Medison, Seoul, Korea) with a high-frequency, linear, 3-12-MHz transducer was used for all procedures. The settings of the US system were optimized for each participant with respect to gain, depth, and focus. First, we identified the VN and traced it throughout the course from level II to level IV to confirm the VN on US and avoid misjudging a small vascular structure as the VN. When necessary, Doppler US was additionally performed to confirm our findings.

After this overview scan, the VN position was evaluated at every level from level II to IV. The usual VN position was defined as a location of the VN posterior or lateral to the $\mathrm{C}-\mathrm{I}$ axis, which was defined as an imaginary axis between the centers of the CA and the IJV on US. The usual location of the VN represents a rarely vulnerable position, because the VN in this position is deep to the CA and the

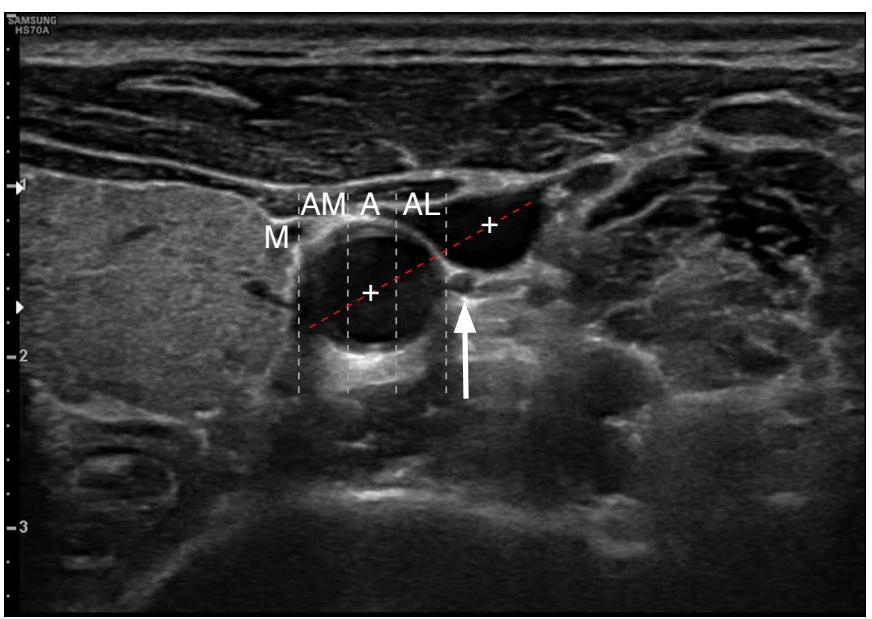

Fig. 2. Usual position of the vagus nerve. The vagus nerve (arrow) is located posterior or lateral to the reference axis, which comprised the carotid artery and the internal jugular vein (red dot line). A variation in position was defined as the vagus nerve being located anterior or medial to the carotid-internal jugular vein axis, and variations were classified into four types-anterolateral (AL), anteromiddle (A), anteromedial (AM), and medial (M)-based on the relative location of the vagus nerve to the carotid artery.
IJV and is protected by these great vessels (Fig. 2). A variation was defined as a location of the VN anterior or medial to the C-I axis, which represents a potentially vulnerable position during surgery or a radiological intervention, as the $\mathrm{VN}$ is located superficial or medial to the CA and the IJV and is not covered by these great vessels. The variations were classified into four types-anterolateral, anteromiddle, anteromedial, and medial-based on the relative location of the VN to the CA. An anterolateral variation was defined as a location of the VN anterior or medial to the $\mathrm{C}-\mathrm{I}$ axis and at the lateral one-third of the CA diameter (Fig. 3A). An anteromiddle variation was defined as a location of the VN anterior or medial to the $C-I$ axis and at the middle one-third of the CA diameter (Fig. 3B). An anteromedial variation was defined as a location of the $\mathrm{VN}$ anterior or medial to the $\mathrm{C}-\mathrm{I}$ axis and at the medial one-third of the CA diameter (Fig. 3C). A medial variation was defined as a location of the $\mathrm{VN}$ anterior or medial to the $\mathrm{C}-\mathrm{I}$ axis and medial to the medial border of the CA (Fig. 3D).

\section{Assessment Parameters and Statistical Analyses}

The primary outcome parameters were the overall prevalence of the VN variation and differences according to side, age, and sex. The secondary outcome parameters were VN variation types, the involved level of the VN variation, and proximity of the VN to the thyroid gland. A vulnerable VN position in thyroid surgery/ radiological intervention was defined as a distance of the $\mathrm{VN}<2$ $\mathrm{mm}$ from the lateral border of the ipsilateral thyroid gland, based on the results of a previous study [2].

According to the study protocol, the patients' age, sex, and the indication for US examination were evaluated. To evaluate differences in VN variations and trends in the prevalence of VN variations according to age, we categorized patients by age into four groups $(<20,20-39,40-59$, and $\geq 60$ years).

SPSS for Windows version 12.0 (SPSS Inc., Chicago, IL, USA) was used to analyze the data. Continuous data are presented as mean \pm standard deviations and were compared between the groups using the independent Student $t$ test. To test the significance of between-group differences in categorical variables, the chi-square test or the Fisher exact test was used. The chi-square test for trend was performed to evaluate the significance of age-related changes for the prevalence of VN variations. Statistical significance was defined as $\mathrm{P}<0.05$, and all $\mathrm{P}$-values are two-sided.

\section{Results}

\section{Baseline Patient Characteristics}

From January 2017 to June 2018, we performed US examinations for 947 patients. Of these patients, 536 patients who met the study's 


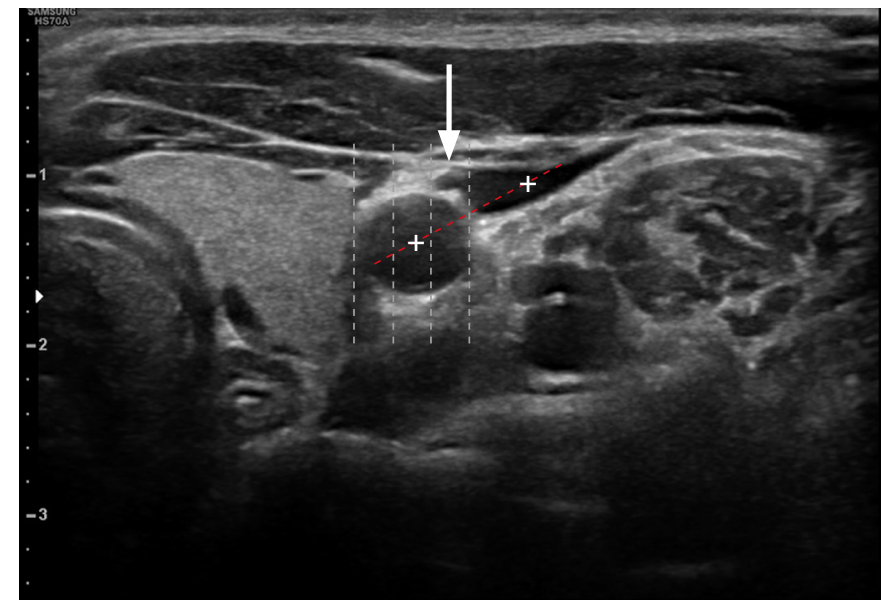

A

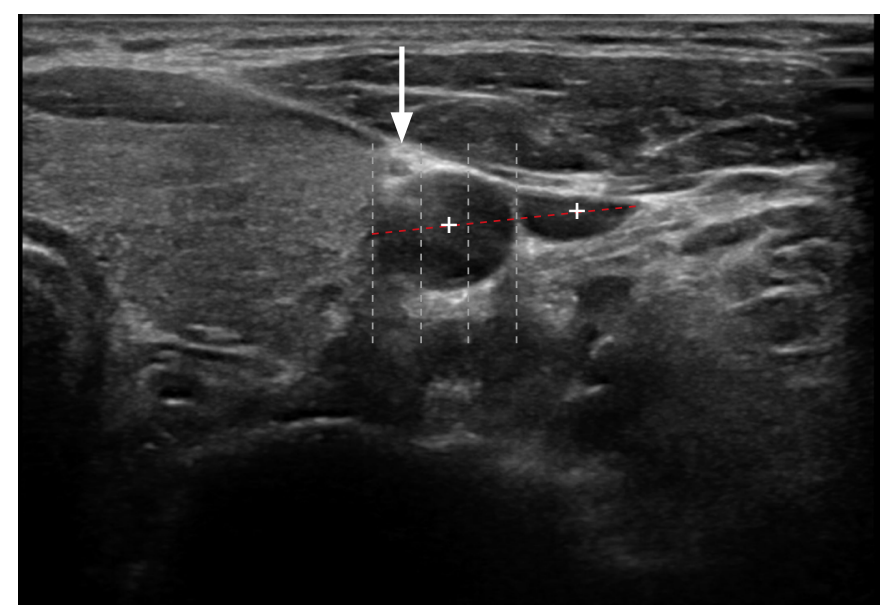

$$
\text { C }
$$

Fig. 3. Types of vagus nerve variation on ultrasonography.

The anterolateral variation, anteromiddle variation, anteromedial variation, and medial variation was defined as the vagus nerve (arrow) being located at the lateral one-third of the carotid artery diameter $(A)$, the vagus nerve (arrow) being located at the middle one-third of the carotid artery diameter (B), the vagus nerve (arrow) being located at the medial one-third of the carotid diameter (C), and the vagus nerve (arrow) being medial to the medial border of the carotid artery (D), respectively.

inclusion and exclusion criteria were enrolled, and the patients' right and left cervical VNs (1,072 in all) were evaluated for the study.

Baseline patient characteristics are presented in Table 1. A total $197(36.8 \%)$ male and 339 (63.2\%) female patients were included, and their mean age was 51.7 years. The number of patients aged $<20,20-39,40-59$, and $\geq 60$ years was $21(3.9 \%), 111(20.7 \%)$, $212(39.6 \%)$, and $192(35.8 \%)$, respectively. The indications for neck US examinations included thyroid/parathyroid lesions in 194 patients (36.2\%), lymphadenopathies in 118 patients (22.0\%), and salivary gland lesions in 104 patients (19.4\%).

\section{Prevalence of Variations in the VN Position by Side and Type} of Variation

For a total of 536 patients, VN variations on either side were

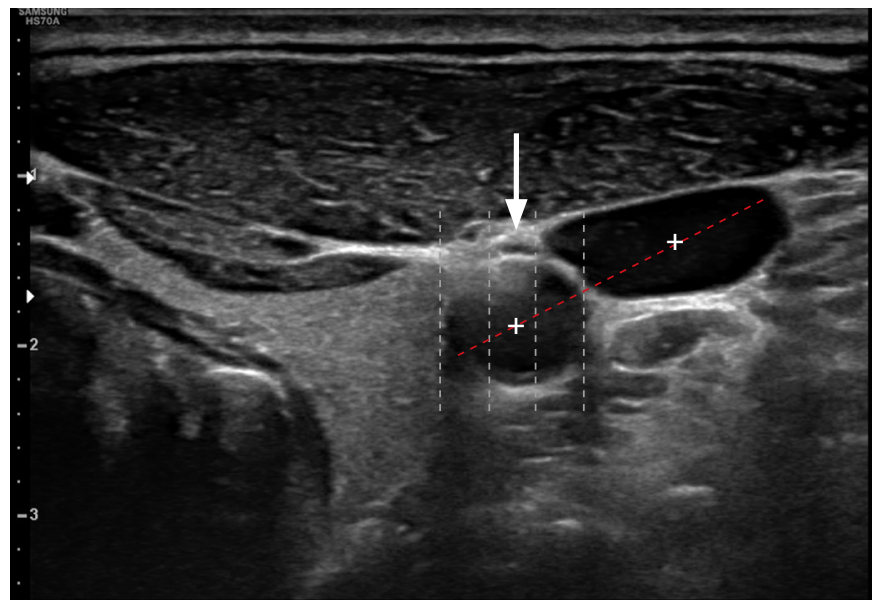

B

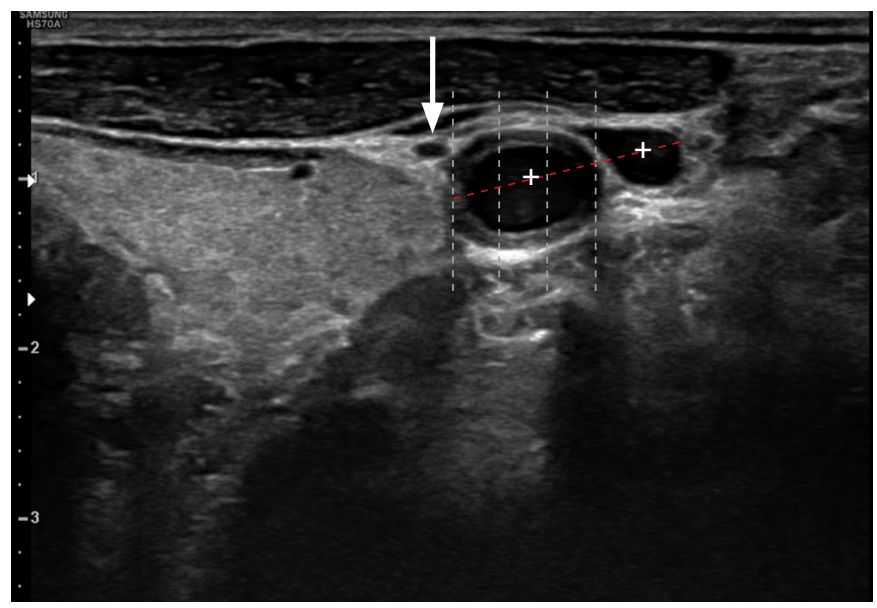

D 
with a right-side VN variation, the variation was identified between levels III and IV and in level IV alone in four (20.0\%) and 16 (80.0\%) patients, respectively. Among the 186 patients with a left-side VN variation, the variation was identified between levels III and IV and in level IV alone in 14 (7.5\%) and 172 (92.5\%) patients,

Table 1. Baseline patient characteristics

\begin{tabular}{lc}
\multicolumn{1}{c}{ Variable } & No. $(\%)(\mathrm{n}=536)$ \\
\hline Sex & $197(36.8)$ \\
Male & $339(63.2)$ \\
Female & \\
Age (year) & $51.7 \pm 17.5$ \\
Mean \pm SD & $21(3.9)$ \\
$<20$ & $111(20.7)$ \\
$20-39$ & $212(39.6)$ \\
$40-59$ & $192(35.8)$ \\
$\geq 60$ & \\
Indication for neck ultrasonography & $194(36.2)$ \\
Thyroid/parathyroid lesion & $118(22.0)$ \\
Lymphadenopathy & $104(19.4)$ \\
Salivary gland lesion & $44(8.2)$ \\
Routine metastatic work-up & $22(4.1)$ \\
Congenital neck mass & $2(0.4)$ \\
Health examination & $52(9.7)$ \\
Other & \\
\hline
\end{tabular}

SD, standard deviation.

Table 2. Prevalence of vagus nerve variations by side and variation characteristics

\begin{tabular}{lccc}
\hline \multicolumn{1}{c}{ Variable } & Right $(\mathrm{n}=536)$ & Left $(\mathrm{n}=536)$ & P-value \\
\hline $\begin{array}{l}\text { Position of the vagus nerve } \\
\text { Normal }\end{array}$ & $516(96.3)$ & $350(65.3)$ & $<0.001$ \\
$\begin{array}{l}\text { Variation } \\
\text { Types }\end{array}$ & $20(3.7)$ & $186(34.7)$ & \\
$\quad$ Anterolateral & $5(25.0)$ & $46(24.7)$ & 0.500 \\
$\quad$ Anteromiddle & $12(60.0)$ & $97(52.2)$ & \\
$\quad$ Anteromedial & $1(5.0)$ & $32(17.2)$ & \\
$\quad$ Medial & $2(10.0)$ & $11(5.9)$ & \\
Level of variation & & & \\
Levels III-IV & $4(20.0)$ & $14(7.5)$ & 0.081 \\
Level IV & $16(80.0)$ & $172(92.5)$ & \\
Proximity to the thyroid gland & & & \\
$<2$ mm & $8(1.5)$ & $50(9.3)$ & $<0.001$ \\
Abutting & $5(0.9)$ & $38(7.1)$ & $<0.001$ \\
\hline
\end{tabular}

Values are presented as number (\%). respectively. The distribution of involved levels was not significantly different between the right and left sides $(P=0.081)$.

Among the 536 right VNs, five $(0.9 \%)$ VNs abutted the right thyroid gland and eight (1.5\%) VNs were $<2 \mathrm{~mm}$ from the lateral border of the right thyroid gland. Of the 536 left VNs, $38(7.1 \%)$ abutted the left thyroid gland and $50(9.3 \%)$ were $<2 \mathrm{~mm}$ from the lateral border of the left thyroid gland. The difference in proximity to the thyroid gland between the right and left sides was statistically significant $(P<0.001)$.

\section{VN Variation according to Patient Demographics}

Table 3 lists VN variations according to patient demographics. Of the 194 patients with a VN variation, $83(42.8 \%)$ and $111(57.2 \%)$ patients were men and women respectively. Among the 342 patients without a VN variation, 114 (33.3\%) and 228 (66.7\%) patients were men and women, respectively. The prevalence of VN variations in male and female patients was $42.1 \%$ and $32.7 \%$, respectively, and the difference was statistically significant $(P=0.029)$. The prevalence of VN variations in patients aged $<20,20-39$, $40-59$, and $\geq 60$ years was $23.8 \%, 22.5 \%, 34.4 \%$, and $47.4 \%$, respectively, and the difference in prevalence among the age groups was statistically significant $(P<0.001)$. In addition, the prevalence of $\mathrm{VN}$ variation showed a significant increasing trend according to increasing age $(P<0.001)$.

\section{Discussion}

This prospective study showed that more than one-third of patients had a variation in the cervical VN position. These variations showed a left-side predominance and increased with age.

Our results showed a similar or higher prevalence of VN variations compared to previous studies ( $36.2 \%$ vs. $5.5 \%-37.8 \%$ ), although the use of the $\mathrm{C}-\mathrm{I}$ axis in our study as a reference rather than the CA

Table 3. Vagus nerve variations according to patient demographics

\begin{tabular}{|c|c|c|c|c|c|}
\hline \multirow[b]{2}{*}{ Variable } & \multirow[b]{2}{*}{ No. } & \multicolumn{2}{|c|}{ No. (\%) } & \multirow{2}{*}{$\begin{array}{l}\text { Prevalence of } \\
\text { variation (\%) }\end{array}$} & \multirow[b]{2}{*}{ P-value } \\
\hline & & $\begin{array}{c}\text { Variation }(+) \\
(n=194)\end{array}$ & $\begin{array}{c}\text { Variation }(-) \\
\quad(n=342)\end{array}$ & & \\
\hline \multicolumn{6}{|l|}{ Sex } \\
\hline Male & 197 & 83 (42.8) & 114 (33.3) & 42.1 & 0.029 \\
\hline Female & 339 & $111(57.2)$ & $228(66.7)$ & 32.7 & \\
\hline \multicolumn{6}{|c|}{ Age (year) } \\
\hline$<20$ & 21 & $5(2.6)$ & $16(4.7)$ & 23.8 & $<0.001$ \\
\hline $20-39$ & 111 & 25 (12.9) & $86(25.1)$ & 22.5 & \\
\hline $40-59$ & 212 & 73 (37.6) & 139 (40.6) & 34.4 & \\
\hline$\geq 60$ & 192 & $91(46.9)$ & $101(29.5)$ & 47.4 & \\
\hline
\end{tabular}


alone was a stricter measure $[1-3,11]$. In fact, the prevalence of VN variations in the cervical region seems to have been underestimated in previous studies because most of those studies employed a retrospective design and the authors used only static US images from a picture archiving and communication system, which did not allow the investigation of the entire course of the cervical VN $[1-3,11]$. In contrast, the present study had a prospective design and the entire course of the cervical VN was assessed by a single operator in each patient; moreover, the study involved a larger number of patients than any previous studies.

In the present study, VN variations were identified primarily in the lower cervical region (level IV). In addition, VN variations were significantly more prevalent in the left side of the neck, with an approximately 10 -fold higher proportion of variations compared to the right side (34.7\% vs. 3.7\%, $\mathrm{P}<0.001)$. These findings might be attributable to the left- to right-side difference in the anatomical course of the VN in the thorax and abdomen; moreover, the VN is the only paired nerve that innervates organs in a markedly asymmetric manner $[13,14]$. In the lower cervical region, the right VN crosses anterior to the right subclavian artery, runs posterior to the superior vena cava, and descends posterior to the right main bronchus. However, the left VN enters the thorax between the left common CA and the left subclavian artery and descends on the aortic arch. In the abdomen, the right VN constitutes the posterior gastric plexus and terminates within the celiac plexus, while the left VN terminates in the anterior gastric plexus, with branches to the stomach, liver, gallbladder, pylorus, and the superior part of the duodenum $[13,15,16]$. Therefore, it seems plausible that the VN shows a difference in course between the left and right sides in the lower cervical region.

On both sides, the anteromiddle type of variation was most common, followed by the anterolateral type. Therefore, in addition to noting the high prevalence of VN variations, head and neck or vascular surgeons should note the possibility of variable VN positions when dissecting the carotid sheath and manipulating the great vessels of the carotid space, particularly when using electrocautery or energy devices, to avoid inadvertent electric or thermal VN injury. If available, it would be ideal for surgeons to use preoperative US to identify the $\mathrm{VN}$ to eliminate risk of inadvertent $\mathrm{VN}$ injury during surgery.

Moreover, our findings showed that $1.5 \%$ of the right VNs and $9.3 \%$ of the left VNs were positioned $<2 \mathrm{~mm}$ from the thyroid gland, including $0.9 \%$ of the right VNs and $7.1 \%$ of the left VNs, which abutted the thyroid gland. These results are comparable to those of a previous study reporting that $13.3 \%$ of VNs in 304 patients were potentially vulnerable during thyroid radiofrequency ablation because of their location [2]. Of all the variation types observed in the present study, the most vulnerable VN positions in the left and right sides of the neck were the anteromedial and medial variations. Therefore, thyroid surgeons and interventional radiologists should be cautious during thyroid surgery or ablation therapy because of the possible vulnerability of the VN, particularly in patients with anteromedial and medial VN variations.

To our knowledge, no study to date has reported age-dependent differences in the prevalence of VN variations based on US findings. Interestingly, we found that the prevalence of VN variation significantly increased from the fifth decade of life onward, with a linear increasing trend according to increasing age $(P<0.001)$. Of particular note, approximately $50 \%$ of patients aged $\geq 60$ years had VN variations, and the prevalence was two-fold higher than that of patients aged $<40$ years $(47.4 \%$ vs. $22.7 \%$ ). This study alone cannot provide a reasonable causal explanation for the increasing prevalence of VN variations with increasing age. However, we may speculate that age-dependent changes in the position of the VN might be associated with senile changes in the structures around the VN, including the sternocleidomastoid muscle, CA, IJV, and carotid sheath, as well as senile changes in the VN $[13,17,18]$. As a person ages, the muscle mass decreases, the fascia loosens, and the great vessels become less taut; these changes may contribute to variability in the VN position $[13,17,18]$.

This study had several limitations. First, the inter-observer reliability of the C-I axis, as a reference to define the VN variation, was not evaluated because this was a prospective cohort study that involved a single US operator. Second, the clinical impact of the VN variation based on the $C-I$ axis was not estimated in patients who underwent neck surgery or ablation procedures. To overcome these limitations, future studies involving multiple assessors for VN variations and evaluating VN injuries following neck surgery or ablation procedures, as a function of $\mathrm{VN}$ variation, should be conducted.

In conclusion, this prospective study demonstrated a somewhat higher prevalence of VN variations than found in previous reports. These variations primarily involved the left VN in the lower cervical region, and an increasing prevalence with increasing age was observed. Therefore, in clinical practice, surgeons and interventional radiologists should consider the presence of variations in the VN position and take steps to avoid inadvertent VN injuries, particularly in the left lower cervical region of older patients.

ORCID: Dongbin Ahn: https://orcid.org/0000-0002-4977-7406; Gil Joon Lee: https:// orcid.org/0000-0002-3344-1879; Jin Ho Sohn: https://orcid.org/0000-0002-74473471; Jeong Kyu Kim: https://orcid.org/0000-0002-5994-1994 


\section{Author Contributions}

Conceptualization: Ahn D. Data acquisition: Ahn D, Lee GJ, Sohn JH, Kim JK. Data analysis or interpretation: Ahn D. Drafting of the manuscript: Ahn D, Kim JK. Critical revision of the manuscript: Ahn D, Lee GJ, Sohn JH. Approval of the final version of the manuscript: all authors.

\section{Conflict of Interest}

No potential conflict of interest relevant to this article was reported.

\section{References}

1. Giovagnorio F, Martinoli C. Sonography of the cervical vagus nerve: normal appearance and abnormal findings. AJR Am J Roentgenol 2001;176:745-749.

2. Ha EJ, Baek JH, Lee JH, Kim JK, Shong YK. Clinical significance of vagus nerve variation in radiofrequency ablation of thyroid nodules. Eur Radiol 2011;21:2151-2157.

3. Park JK, Jeong SY, Lee JH, Lim GC, Chang JW. Variations in the course of the cervical vagus nerve on thyroid ultrasonography. AJNR Am J Neuroradiol 2011;32:1178-1181.

4. Kakisis JD, Antonopoulos CN, Mantas G, Moulakakis KG, Sfyroeras $G$, Geroulakos G. Cranial nerve injury after carotid endarterectomy: incidence, risk factors, and time trends. Eur J Vasc Endovasc Surg 2017:53:320-335.

5. Thirumala PD, Kumar H, Bertolet M, Habeych ME, Crammond DJ, Balzer JR. Risk factors for cranial nerve deficits during carotid endarterectomy: a retrospective study. Clin Neurol Neurosurg 2015;130:150-154.

6. Larsen MH, Lorenzen MM, Bakholdt V, Sorensen JA. The prevalence of nerve injuries following neck dissections: a systematic review and meta-analysis. Dan Med J 2020;67:A08190464.

7. Ben Hamou A, Ghanassia E, Espiard S, Abi Rached H, Jannin A, Correas JM, et al. Safety and efficacy of thermal ablation (radiofrequency and laser): should we treat all types of thyroid nodules? (dagger). Int J Hyperthermia 2019;36:666-676.

8. Hahn SY, Shin JH, Na DG, Ha EJ, Ahn HS, Lim HK, et al. Ethanol ablation of the thyroid nodules: 2018 Consensus Statement by the Korean Society of Thyroid Radiology. Korean J Radiol 2019;20:609620.

9. Goyal RM, Jonklaas J, Burman KD. Management of recurrent cervical papillary thyroid cancer. Endocrinol Metab Clin North Am 2014;43:565-572.

10. Paz-Fumagalli R, Li X, Smallridge RC. Ethanol ablation of neck metastases from differentiated thyroid carcinoma. Semin Intervent Radiol 2019:36:381-385.

11. Chen HH, Chen TC, Yang TL, Wang CP. Transcutaneous sonography for detection of the cervical vagus nerve. Ear Nose Throat J 2021;100:155-159.

12. Arya $R$, Antonisamy $B$, Kumar $S$. Sample size estimation in prevalence studies. Indian J Pediatr 2012;79:1482-1488.

13. Pelz JO, Belau E, Henn P, Hammer N, Classen J, Weise D. Sonographic evaluation of the vagus nerves: Protocol, reference values, and side-to-side differences. Muscle Nerve 2018;57:766771.

14. Curcean AD, Rusu GM, Dudea SM. Ultrasound appearance of peripheral nerves in the neck: vagus, hypoglossal and greater auricular. Med Pharm Rep 2020;93:39-46.

15. Sibilla L, Agarwal N. Cranial nerve X: vagus. In: Agarwal N, Port JD, eds. Neuroimaging: anatomy meets function. Cham: Springer, 2018;211-214.

16. Shahid Z, Burns B. Anatomy, abdomen and pelvis, diaphragm. Treasure Island, FL: StatPearls Publishing, 2017.

17. Rebeiz JJ, Moore MJ, Holden EM, Adams RD. Variations in muscle status with age and systemic diseases. Acta Neuropathol 1972;22:127-144.

18. Schmidt-Trucksass A, Grathwohl D, Schmid A, Boragk R, Upmeier C, Keul J, et al. Structural, functional, and hemodynamic changes of the common carotid artery with age in male subjects. Arterioscler Thromb Vasc Biol 1999;19:1091-1097. 